\title{
Mitochondrien-Veränderungen pflanzlicher Zellen bei Störung von Atmungsprozessen
}

\author{
M. Wrischer und Z. DevidÉ \\ Aus dem Laboratorium für Elektronenmikroskopie, Biologische Abteilung, \\ Institut "Rudjer Bošković", Zagreb, Jugoslawien \\ (Z. Naturforschg. 20 b, 260-263 [1965] ; eingegangen am 6. November 1964)
}

\begin{abstract}
Ultrathin sections through the root tips of some plants (Sinapis alba, Allium cepa), which were held under anoxic conditions or treated with some inhibitors of respiratory enzymes $\left(\mathrm{NaN}_{3}, \mathrm{KCN}\right)$, showed in the electron microscope striking elongations (i.e. enlargements) and peculiar deformations of certain mitochondria. On treated living cells it could be shown by means of the light microscope that the described mitochondrial changes are easily reproducible and that in reversibly damaged cells the elongated mitochondria do not restore their normal shape and size, but seem to degenerate gradually. The described changes are considered to be a consequence either of an irreversibly inhibited mitochondrial division or of a pathobiotic structural adaptation.
\end{abstract}

Während elektronenmikroskopischer Untersuchungen über Veränderungen des endoplasmatischen Reticulums, die bei Pflanzen als Folge von Störungen der Zellatmung (bei Anoxie oder nach Einwirkung von Natriumazid u. a. Enzyminhibitoren) entstehen ${ }^{1,2}$, konnte gelegentlich das Vorkommen sehr auffallend vergrößerter Mitochondrien festgestellt werden. Systematisch durchgeführte Untersuchungen bestätigten diese Beobachtungen und ermöglichten das Auffinden von experimentellen Bedingungen, bei denen das Auftreten derartig veränderter Mitochondrien mit großer Wahrscheinlichkeit reproduzierbar ist. Nachdem die mit dem Lichtmikroskop zunächst nur gelegentlich und fragmentär unterstützten elektronenmikroskopischen Ergebnisse ${ }^{3}$ durch ausreichende lichtmikroskopische Untersuchungen ergänzt und in Einklang gebracht werden konnten, sollen hier alle bisher erhaltenen Resultate in Gänze mitgeteilt werden.

\section{Material und Methoden}

Die grundlegenden Versuche wurden an Wurzelspitzen $2-3$ Tage alter Senfkeimlinge (Sinapis alba L.) durchgeführt. Neben Senfkeimlingen wurden gelegentlich auch Wurzeln und (für lichtmisroskopische Zwecke) isolierte Epidermisstücke der Küchenzwiebel (Allium сера L.) verwendet.

1 M. Wrischer, Naturwissenschaften 47, 521 [1960].

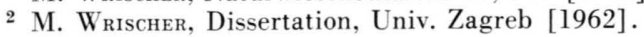

Die Störung der Zellatmung wurde durch Anoxie (durchgekcchtes Leitungswasser ohne Luftzutritt, 2 bis mehrere Stdn. lange) oder durch Einwirkung von Inhibitoren der Atmungsenzyme $\left(10^{-4}-m\right.$. Lösung von Natriumazid oder Kaliumcyanid in Leitungswasser bei $12-15$ Stdn. langer Einwirkung) hervorgerufen, wobei die überwiegende Mehrzahl der Versuche mit der Natriumazid-Lösung durchgeführt wurde.

Die günstigen Stadien wurden unter einer Bino-Lupe herausgesucht und für die entsprechenden mikroskopischen Untersuchungen verwendet. Die Erfahrung hat nämlich gezeigt, daß die zu untersuchenden Veränderungen an Mitochondrien gerade in jenem Zeitintervall auftreten, in dem die Wurzelspitzen durch die pathobiotisch bedingten Zell- bzw. Plasmaveränderungen (hauptsächlich Vakuolisation) undurchscheinend zu werden beginnen.

Für elektronenmikroskopische Zwecke wurde das Material teils mit Osmiumtetroxyd (1-proz. gepufferte Lösung, $\quad p_{\mathrm{H}}=7,2-7,4$, mit Saccharosezusatz bis 0,15-m.; Dauer 2-3 Stdn.) teils aber mit Kaliumpermanganat (5-proz. Lösung in dest. Wasser; 2 Stdn.) fixiert. Osmium-fixiertes Material wurde in Methacrylatgemisch eingebettet, Permanganat-fixiertes dagegen in Araldit. Die Schnitte wurden mit dem ReichertUltramikrotom (nach S i t te) hergestellt und mit dem elektrostatischen Mikroskop KM-4 der Firma Trüb, Täuber \& Co. untersucht und aufgenommen.

Lichtmikroskopische Beobachtungen wurden ausschließlich am lebenden Material durchgeführt, wobei kontinuierliche Beobachtungen an gewählten Zellen bis über $8 \mathrm{Stdn}$. lang durchgeführt wurden. Hierzu wurden mit einwandfrei scharfen Rasierklingen hergestellte

3 Die ersten Ergebnisse wurden auf der Tagung für Elektronenmikroskopie in Zürich, 23.-26. September 1963 (Autorenreferate S. 60 und Mikroskopie [Wien] 19, 43 [1964]), kurz mitgeteilt. 

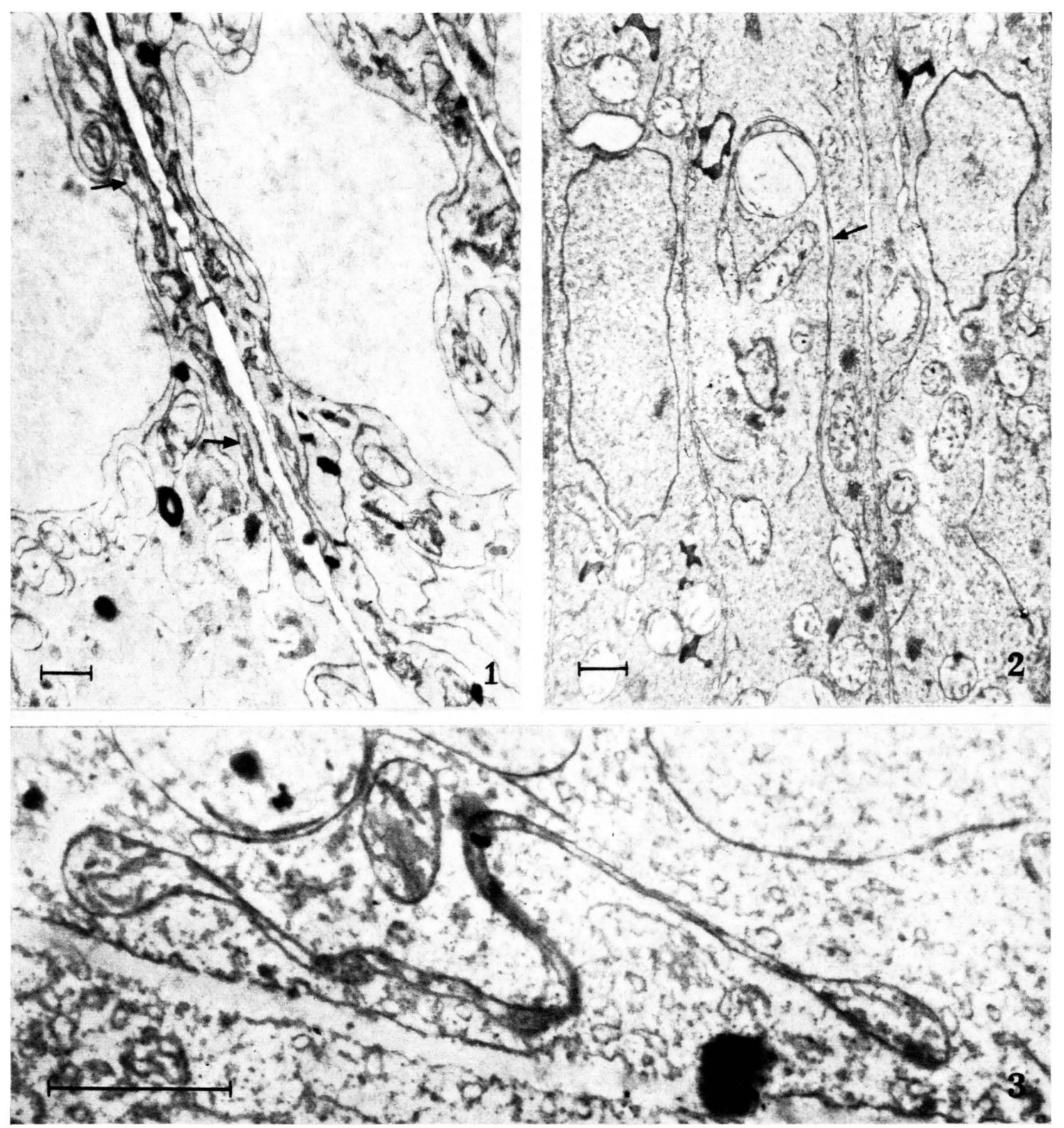

Abbn. 1-3. Sinapis alba, Wurzelspitze, $\mathrm{NaN}_{3}$-Behandlung $\left(10^{-4}-m ., 14-15\right.$ Stdn. lang). Elektronenmikroskopische Aufnahmen.

Abb. 1. Einzelne vergrößerten Mitochondrien ( $\uparrow) . \mathrm{KMnO}_{4^{-}}$ Fixierung, $7000: 1$.
Abb. 2. Ein beträchtlich vergrößertes Mitochondrium ( $\uparrow)$, gewunden um die Proplastide (Länge $=13 \mu$ ). $\mathrm{OsO}_{4}$-Fixierung, $7000: 1$.

Abb. 3. Ein vergrößertes Mitochondrium (Länge $=7 \mu$ ). $\mathrm{OsO}_{4}$-Fixierung, $26000: 1$. 

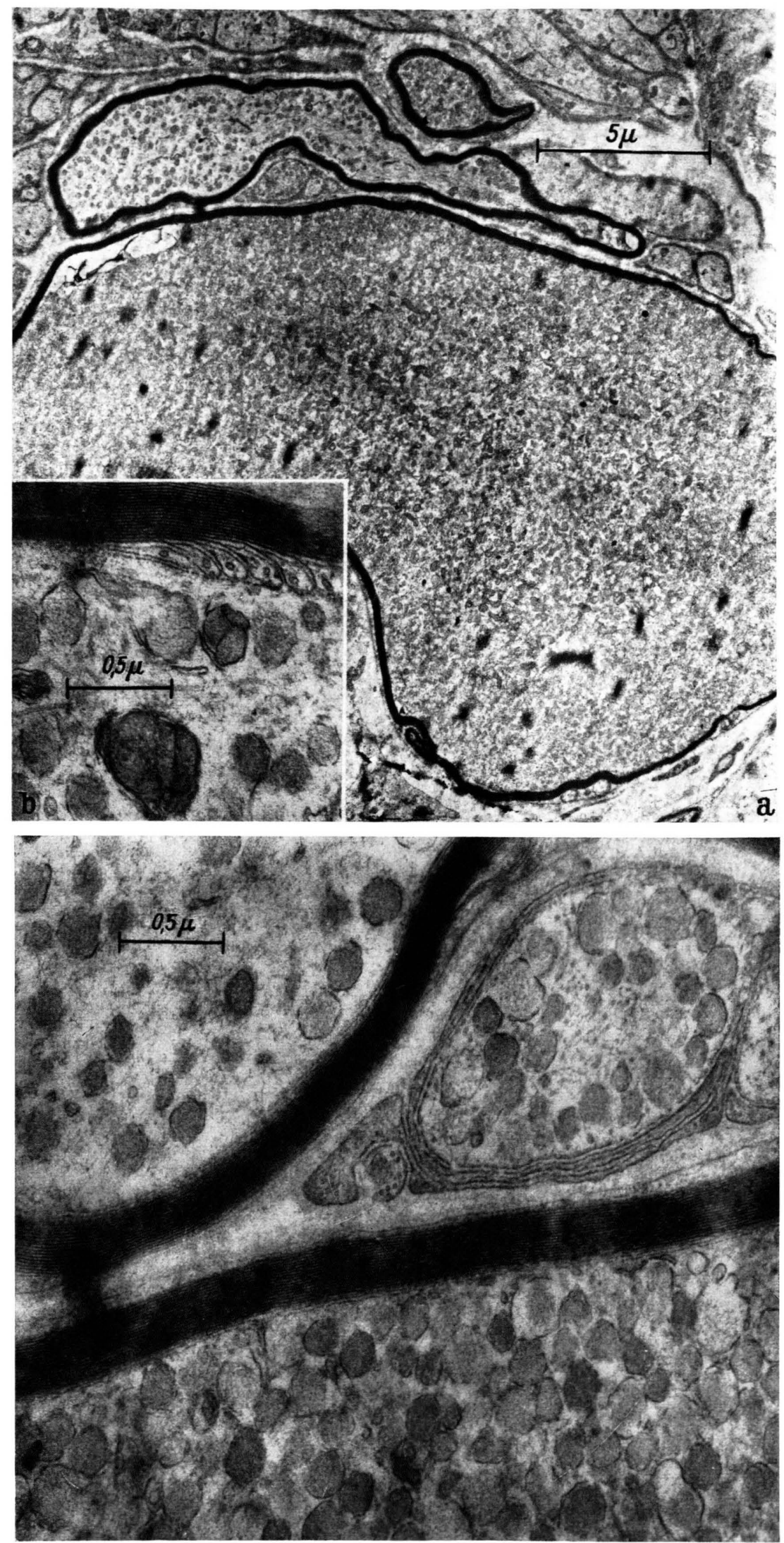

Abb. 1. a) Myelinisierte Axone mit EG aus dem distalen Stiel, 24 Stdn. nach Koagulation. Arch. Nr. 2644/64; el.-opt. Vergr.: 2500-fach. b) Ausschnitt aus einem EG-haltigen Axon, dessen Myelinlamellen ähnlich wie an Schnürringen aufgeblättert sind. Arch. Nr. 2364/64; el.-opt. Vergr.: 10000 -fach.
Abb. 2. Anschnitt von zwei myelinisierten Axonen mit EG, in der Mitte ein Axon mit EG, das lose angeordnete Membranduplikatoren erkennen läßt. Arch. Nr.: 2646/64; el.-opt. Vergr.: 10000 -fach. 
und nicht zu dünne Freihandschnitte durch behandelte sowie unbehandelte Wurzeln bei Verwendung stärkster Optik in Deckglaspräparaten untersucht. Die Untersuchungsflüssigkeit unter dem Deckglas (Azid- bzw. Cyanidlösung oder reines sauerstoffreiches Leitungswasser) wurde nach Bedarf von Zeit zu Zeit erneuert. Die innere Epidermis der Zwiebelschuppen wurde mit Hilfe der Infiltrationsmethode vorsichtig wegpräpariert, sonst aber völlig gleichartig wie die Wurzelschnitte behandelt und untersucht.

\section{Elektronenmikroskopische Beobachtungen}

Bei etwa $40 \%$ * der eingebetteten Wurzelspitzen konnten an Ultradünnschnitten in den Zellen der dritten und vierten Zellschicht (gezählt von außen nach innen einschließlich die Rhizodermis) auffallend vergrößerte Mitochondrien von merkwürdiger Gestalt aufgefunden werden. Während unter normalen Bedingungen in Dünnschnitten die Mitochondrien dieser Zellen annähernd kreisförmig oder nur schwach verlängert erscheinen (höchstens $0,65 \cdot 2 \mu$ ), nehmen im behandelten Material einzelne Mitochondrien an der im Schnitt gemessenen Länge sehr zu, und zwar meistens bis auf $5-10 \mu$, manchmal sogar $20 \mu$.

Die durchschnittliche Gestalt dieser Mitochondrien konnte aus den elektronenmikroskopischen Bildern der in zwei gegenseitig normalen Richtungen gewonnenen Ultradünnschnitten ermittelt werden, besonders wenn diese von demselben Präparatblock stammten. In Längsschnitten findet man vorwiegend hantelförmige Figuren vor, die an besonders heftige Teilungsstadien erinnern. Sie sind vermutlich aus räumlichen Gründen (die Zellen sind kurz zylindrisch!), vorwiegend parallel zur Querschnittebene, also normal zur Längsachse der Zellen und der Wurzel, orientiert und hierbei verschiedenartig gebogen (Abbn. $1^{* *}, 2,3$ ). Die Breite der Endpartien beträgt im Schnitt $0,35-0,8 \mu$, jene der mittleren Partien jedoch bedeutend weniger, oft nur $0,05 \mu$, manchmal sogar noch weniger. In Querschnitten erscheinen die veränderten Mitochondrien meistens als kürzere, aber einige $\mu$ breite Gebilde. Aus den Bildern der beiden Schnittrichtungen ergibt sich, daß diese Mitochondrien im Durchschnitt eine Form von länglichen, am Rande verdickten scheiben- oder bandartigen Bildungen haben müssen, die zuweilen gebogen oder selbst

* Diese Zahl entspricht der Wahrscheinlichkeit, mit der in den auf die angegebene Weise in toto fixierten Wurzeln das Stadium der Mitochondrien-Veränderungen angetroffen wurde. becherförmig gekrümmt sind. Hufeisen- oder gar ringförmige Figuren in den Schnitten bestätigen dies sehr gut.

\section{Lichtmikroskopische Beobachtungen}

Die beschriebenen elektronenmikroskopisch festgestellten Veränderungen an Mitochondrien konnten wir mit dem Lichtmikroskop auch an lebenden Präparaten nachweisen. In richtig hergestellten Handschnitten durch behandelte, aber noch lebende Wurzelspitzen, konnten im gewöhnlichen Hellfeld an geeigneten Präparaten die vergrößerten Mitochondrien recht deutlich stundenlang kontinuierlich beobachtet werden. Zellen, die für die lichtmikroskopischen Beobachtungen am günstigsten waren, waren Zellen aus der Streckungszone, während die aus der embryonalen Zone stammenden Zellen, die für das Elektronenmikroskop am geeignetsten waren, aus optischen Gründen (Undurchsichtigkeit der Stellen infolge des inhaltsreichen Plasmas der Zellen) dem Lichtmikroskop nicht leicht zugänglich waren. Vergleichende Untersuchungen zeigten jedoch eindeutig, daß es sich um identische Organellen-Veränderungen handelt. Isolierte und entsprechend behandelte Stücke der Zwiebelepidermis zeigten genau die gleichen Erscheinungen.

Die Untersuchungen der mit $\mathrm{NaN}_{3}$ oder KCN behandelten Wurzeln sowie Zwiebelepidermen haben gezeigt, $d a ß$ in den Zellen mit eben noch vorhandener Plasmaströmung sehr häufig neben normalen Mitochondrien auch eine Anzahl veränderter Mitochondrien anzutreffen ist. Die Form dieser vergrößerten Mitochondrien ist recht verschieden (Abb. 4). Diese Formen konnten mit den elektronenmikroskopischen Bildern ohne weiteres völlig in Einklang gebracht werden. Bei Mitochondrienformen, deren mittlere Partien nach elektronenmikroskopischen Untersuchungen submikroskopisch dünn ausgezogen waren, konnte eine völlig richtige Abbildung wegen Unterschreitung der Auflösungsgrenze sowie zu geringen Lichtbrechungs-Unterschieden natürlich im Lichtmikroskop nicht erwartet werden.

Die Meßresultate stimmten ebenfalls mit den elektronenmikroskopischen gut überein. Mitochondrien mit einer Länge von etwa $5-11 \mu$ waren sehr

** Abbn. 1-3 s. Tafel S. 260 a.

1. Frauenklinik und Hebammencohcte der Universität Münche:

München 15, Maistraße 11 Bucherel 


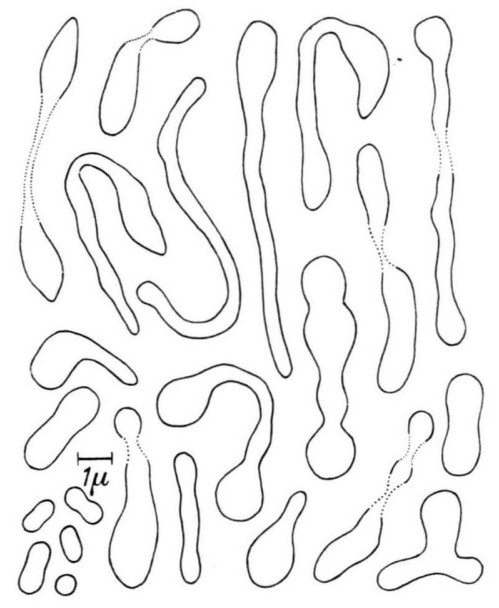

Abb. 4. Umrisse einiger vergrößerter Mitochondrien unter dem Lichtmikroskop. Zum Vergleich links unten fünf normale Mitochondrien. (Sinapis alba, Wurzelspitzen behandelt mit $\mathrm{NaN}_{3}$ und unbehandelte Kontrolle. Ölimmersion, Freihandzeichnung nach den Frischpräparaten auf Grund von Vermessungen.)

häufig. Formen von etwa $20 \mu$ wurden im Lichtmikroskop zwar nicht bemerkt, was jedoch zu erwarten war, da so extrem lange Formen in der mittleren Partie stets unter $500 \AA$, also weit unter der Auflösungsgrenze des Lichtmikroskopes, dünn ausgezogen waren. Das größte im Lichtmikroskop gemessene Mitochondrium hatte aber doch eine Länge von $15 \mu$.

Sehr wichtig waren die lichtmikroskopischen Untersuchungen für die Prüfung der Reversibilität der beobachteten Mitochondrien-Veränderungen. Wäscht man die Azidlösung bzw. Cyanidlösung aus dem Präparat rechtzeitig heraus und bringt in entsprechenden Zeitabständen frisches Leitungswasser hinzu, so erholen sich die Zellen recht bald (innerhalb einiger Min.) wieder und es tritt eine lebhafte Plasmaströmung ein. Es wurde festgestellt, daß innerhalb 7 - 8 Stdn. nach Beendigung der Behandlung (d. h. nach dem Zusetzen von reinem Leitungswasser) die vergrößerten Mitochondrien weiterhin wesentlich unverändert bleiben. Es konnte kein Anzeichen dafür gefunden werden, daß die Mitochondrien wieder ihre normale Form annehmen würden. Es scheint vielmehr, daß bei den vergrößerten Mitochondrien weitere Deformierungen in Form von Aufblähungen auftreten, bis endlich diese geschädigten Organellen wahrscheinlich irgendwie zugrundegehen. Da die

${ }^{4}$ L. Geitler, Protoplasma 27, 534 [1937].

5 M. N. Končalova, Biol. Plantarium [Praha] 4, 170 [1962].
Anzahl solcher veränderten Mitochondrien relativ gering ist, ist ihr Schicksal offensichtlich für das Leben der Zelle nicht ausschlaggebend, denn daß solche Zellen durch die beschriebene Azid- oder Cyanid-Einwirkung nur reversibel geschädigt werden, steht auf Grund vorliegender lichtmikroskopischen Untersuchungen außer jedem Zweifel.

\section{Diskussion}

Keulenförmige Deformationen der Mitochondrien, allerdings ohne einer auffallenden Größenzunahme, hat Geituer ${ }^{4}$ bei Diatomeen bei längerem Verweilen unter dem Deckglas (Anoxie) bereits lichtmikroskopisch festgestellt. Končalova ${ }^{5}$ beobachtete eine Verlängerung der Mitochondrien in Wurzelhaaren der Gerste und in Zwiebel-Epidermiszellen, die in einer $\mathrm{CO}_{2}$-Atmosphäre gehalten wurden. Ähnliche Veränderungen wurden lichtmikroskopisch auch bei Mitochondrien tierischer Zellen festgestellt ${ }^{6}$.

In den letzten Jahren sind einige elektronenmikroskopische Arbeiten erschienen, die mehr oder weniger ähnliche Mitochondrien-Deformationen wie die hier beschriebenen, die nach Einwirkung verschiedener Faktoren entstehen, behandeln. Von diesen sollen hier nur einige berücksichtigt werden.

Zunächst wären die Untersuchungen von $\mathrm{S}_{\mathrm{CHNEPF}}{ }^{7}$ an Drüsenzellen von Drosophyllum, die mit Cyanidbzw. Arsenitlösungen behandelt wurden, zu erwähnen. Die Beschreibung und die veröffentlichten Abbildungen lassen jedoch keine nähere morphologische Ähnlichkeit seiner Befunde mit den von uns beobachteten Vorgängen vermuten. Zwar konnten wir an unseren Abbildungen bei einzelnen Mitochondrien, die nicht sehr vergrößert waren, eine anscheinende Parallelisierung von der an Zahl zugenommenen Differenzierungen der Innenmembran (Cristae?) vermuten, wie sie Schnepf sehr stark ausgeprägt in seinem Material beobachtet hat, verfügen aber wegen des zu niederen Auflösungsvermögens des vorhandenen Instrumentes über keine entsprechenden sicheren Bildbelege. Merkwürdige Verlängerungen und Deformierungen der Mitochondrien, wie wir sie bei unseren Objekten sowohl licht- als auch elektronenmikroskopisch einwandfrei nachweisen und reproduzieren konnten, hat Schnepf bei Drosophyllum jedoch nicht beobachtet.

6 A. B. Novikoff, Mitochondria (Chondriosomes), in: J. Brachet and A. E. Mirsky, ed., The Cell, Vol. II, Acad. Press, New York-London 1961.

7 E. Schnepf, Flora [Jena] 153, 23 [1963]. 
Die in der vorliegenden Arbeit beschriebenen Mitochondrien-Veränderungen stimmen in morphologischer Hinsicht dagegen weitgehend mit den Ergebnissen der Untersuchungen von Reynolds ${ }^{8}$ überein, der bei Ratten in den Zellen der Leber eine sehr ähnliche Vergrößerung und Deformierung der Mitochondrien als Folge von Giftwirkungen des Tetrachlormethans festgestellt hat. Die Ähnlichkeit besteht besonders in der Ausbildungsweise der sehr verdünnten mittleren Partien der Mitochondrien, in den sogenannten "regions of attenuation“. REynoLDs beobachtet solche vergrößerten Formen als Folge einer Mitochondrien-Verschmelzung oder einer Synthese von zusätzlichem Mitochondrien-Material. Einige der gefundenen Stadien sprechen nach seiner Meinung sehr für ein Vorkommen von Mitochondrien-Verschmelzung, doch konnte er eine bemerkbare Abnahme der Mitochondrienzahl in den Zellen nicht feststellen. Dagegen konnte er auf Grund des vorhandenen Tatsachenmaterials die Möglichkeit einer Synthese neuer Mitochondriensubstanz nicht ausschließen.

Bei unseren Untersuchungen konnten wir feststellen, daß - sofern es sich nicht um sehr späte Stadien der Nekrobiose von irreversibel geschädigten Zellen handelte - die vergrößerten Mitochondrien stets kontinuierliche, also nicht unterbrochene oder zerrissene Außenmembranen hatten und daß ebenso, gemäß dem unveränderten Elektronen-Streuungsvermögen, die Dichte ihrer Matrix sich nicht geändert hat. Wir betrachten es deshalb als eine Tatsache, daß es parallel mit der Vergrößerung der Mitochondrien zu einer Zunahme der Mitochondriensubstanz (d. h. der Membranen und der Matrix) kommen muß. Dies ist nur durch zwei Vorgänge denkbar: entweder durch Fusion einzelner Mitochondrien oder durch Neubildung mitochondrialer Substanz unter gleichzeitiger Volumzunahme des Mitochondriums. Eine Fusion der Mitochondrien ist aus der Literatur bekannt ${ }^{6}$. Im vorliegenden Fall erscheint sie aber so-

-8 E. S. Reynolds, J. Cell Biology 19, 139 [1963]. wohl auf Grund licht- wie elektronenmikroskopischer Beobachtungen als sehr unwahrscheinlich. Es ist auch rein theoretisch kaum denkbar, daß die Mitochondrien durch sehr lange faden- oder blattförmige Fortsätze auf weite Entfernung hin verschmelzen würden. Auch wurden Stadien, die einer Verschmelzung durch gegenseitige Berührung zweier benachbarter Mitochondrien entsprechen würden, nicht gesehen. Aus diesen Gründen glauben wir die Möglichkeit einer Mitochondrienfusion ausschließen zu dürfen.

Es bleibt also nur die Möglichkeit einer Mitochondrien-Vergrößerung durch Substanzneubildung übrig. Hier müssen wir jedoch mindestens zwei Möglichkeiten berücksichtigen. Im ersten Falle würde die Neubildung mitochondrialer Substanz normal vor sich gehen, die Vergrößerung der Mitochondrien würde aber durch eine Teilungshemmung zustande kommen. Im zweiten Fall müßte man eine pathologisch bedingte, abnormale Bildung neuer mitochondrialer Substanz voraussetzen, die durch die Störung der Atmungsprozesse hervorgerufen wäre und die die durch den Enzyminhibitions-Vorgang funktionslos gewordenen Mitochondrien-Elemente ersetzen würde, wie dies u. a. auch $\operatorname{SchepF}^{7}$ sowie Wilson und LEDUC ${ }^{9}$ annahmen. Sollte dies zutreffen, so wäre noch zu erklären, warum so eine Vergrößerung nur einzelne, nicht aber alle Mitochondrien erfahren. Hier erscheinen wieder zwei Deutungen möglich. Es ist nämlich denkbar, daß es sich entweder um funktionell verschiedenartige Mitochondrien handelt oder aber, daß eine zufallsmäßige Verteilung der Schädigungswirkung an einzelne Mitochondrien vorliegt.

Die Irreversibilität der Mitochondrien-Vergrößerung in den durch die Störung von Atmungsprozessen reversibel geschädigten Zellen spricht dafür, daß, abgesehen davon, ob es sich um inhibierte Teilungsstadien oder um eine pathologisch bedingte Neubildung der Mitochondriensubstanz handelt, die Mitochondrien-Vergrößerung einen nekrobiotischen Charakter hat bzw. einen Teilprozeß der Zellnekrobiose darstellt.

9 I. W. Wilson and F. H. Leduc, J. Cell Biology 16, 281 [1963]. 